\title{
The design and implementation of forecasting and application system for wind power
}

\author{
Ma Weiqing, Jia Zhiyi, Bai Zhiguo \\ The Department of Information Technology \\ Shanxi Yangquan Electric Power Corporation \\ Yangquan, China \\ mwq2223@sina.com.
}

\author{
MU Zhaoxi \\ Huada Tianyuan (Beijing) Electric Porwer Tecnaology \\ Co., LTD \\ Smart Grid Department \\ Beijing, China \\ mzx0521@126.com.
}

\begin{abstract}
With the extensive use of wind power, how to predict wind power effectively, to ensure the security, stability and economic operation of grid-connected wind power becomes a serious problem for the electronic companies. Based on the analysis about the status of wind power forecasting and application, the article designs a set of wind power forecasting and application system aimed at the security, stability of gridconnected wind system, which is described from the ideas of construction, software architecture, and development of hardware.
\end{abstract}

Keywords-wind power, power, forecasting, power grid

\section{INTRODUCTION}

Over the years, with the depth study of technology about new energy power generation, wind power is widely used in the field of new energy power generation because of its rich resources, non-pollution, renewable and other features. However, even though wind power provides clean energy, it also brings some new problems to the traditional power system operation. The driving force behind the wind turbine is from the wind flowing through the blower, and the output power of the blower is mainly determined by wind speed, air density and so on, and wind speed is a volatile variable, which would change a lot in a short period, and this change is intermittent, irregular, which will lead to rapid fluctuations in output power of the blower. Because of the random fluctuation, when wind power connected to network in a large scale, the operating personnel cannot conduct electricity planning, and cannot guarantee that the station can just give enough power output when it is needed, which making the optimization management of wind power becomes very difficult, and also impact on the stability, controllability and economy of the power grid.

The mainly effects on the traditional power grid brought by large-scale grid-connected wind power reflected in:

1) Large-scale grid-connected wind power impacts on system peak load dispatch. Since wind power is an "intermittent" power generation, and the correlation between the output and the rule of load changing is not very strong, large-scale grid-connected wind power has laid enormous pressure on peak load dispatch of traditional grid system.

2) Access of large-scale wind power impacts on the operational control. For the sake of random fluctuations in wind power, it is difficult for the dispatch departments to develop and implement accurate projects as usual.

3) Access of large-scale wind power impacts on economic performance. For the sake of random fluctuations in wind power, and the little correlation between the output and the rule of load changing, the power grid needs to increase the corresponding spinning reserve capacity continually according to the increase electricity.

\section{RESEARCH STATUS}

In wind power developed countries, the study of wind power forecasting technology has been carried out for nearly 20 years. The first research unit was Denmark's Risoe National Laboratory, and then Germany had also carried out in-depth studies. There are many commercial forecasting system has been put into operation although the used methods are vary. Wind power forecasting methods are divided into the basic two types -- physical method and mathematical statistical method [1-4].

Physical method obtains weather data such as wind speed, wind direction, pressure, temperature etc. from forecasting results of numerical weather prediction system. Based on the information about contours, roughness, obstacles, temperature stratification around the wind-farm, the system can calculate the wind speed, wind direction, pressure, and temperature etc. at the height of wind turbine hub, whose method is similar to WAsP. And then the system can calculate the output power in wind farm according to the power curve. For example: Prediktor developed by Denmark's Risoe National Laboratory and Ewind developed by TrueWind in the U.S.

Statistical method identifies the relationship between weather conditions and wind farm output according to historical statistical data, rather than considers the physical process of the change of wind speed. And then the system predicts the output power in wind farm according to the measured data and numerical weather prediction data. The commonly used forecast methods include time series, BP Neural Network, RBF Neural Networks and SVM (Support Vector Machines) and so on, such as autoregressive-based WPPT model developed by Technical University in Denmark, ANN-based (Artificial Neural Network) WPMS system developed by ISET in German. 


\section{FORECASTING METHODS FOR WIND POWER}

\section{A. Data preparation}

The data used in the modeling of wind power forecasting system should include historical data of power, wind tower, numerical weather forecasting, and the information about wind turbines and their running status, and topography.

1) Historical data of power in wind-farm:Historical data of power in wind-farm cannot less than $1 \mathrm{a}$, and the time resolution cannot less than $5 \mathrm{~min}$.

2) Historical data of wind tower:Location of wind towers should within $5 \mathrm{~km}$ around the wind farm, the data should include wind speed, direction, air temperature, and barometric pressure and so on in $10 \mathrm{~m}, 70 \mathrm{~m}$ and above at least. And time resolution cannot less than $10 \mathrm{~min}$.

3) Historical numerical weather forecasting:Corresponding to historical data of power, time resolution of historical numerical weather forecasting is $15 \mathrm{~min}$, and it should include parameters such as wind speed, direction, temperature, pressure and humidity and so on in three different layers at least.

4) Information about wind turbines:The information should include the type of turbines, the capacity of the single one, hub height, rotor diameter, power curve, time of grid-connected location (longitude and latitude), etc.

5) Information about running status of wind turbines and the wind farm:The information should include records about breakdown and the artificial downtime of the turbines, wind power capacity and power rationing records.

\section{B. Forecasting methods}

Considering the factors in wind power prediction system such as modeling difficulty, computational speed, the demand of historical data and accuracy and so on, the wind power forecasting system in Ningxia power grid adopts BP neural network (BackPropagation Neural Network) method for wind power prediction. As shown in Figure 1, BP neural network refers to multi-layer forward neural network based on back propagation algorithm of the error, using a teacher training methods.

Characteristics of BP neural network are as follows:

1) The network is able to approximate any non-linear by arbitrary precision, in order to achieve complex systems modeling;

2) The network can learn and adaptive to unknown information, so that if the system is changed, the forecast effect can be changed through the revision network joint value;

3) The distributional information storage and the processing structure, have certain fault tolerance, therefore the formed system has the good robustness;

4) The model structure of multi-input, multi-output is suitable for handling complex problems.

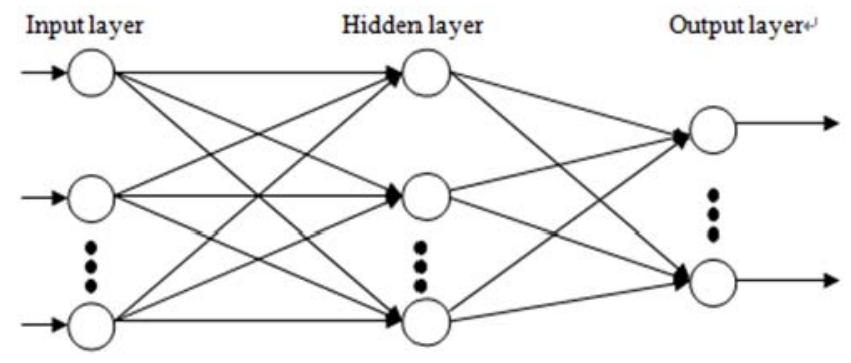

Figure 1. Structure of BP neural network

The trained neural network has good generalization ability to identify unfamiliar data. Therefore, the introduction of artificial neural networks for forecasting of wind speed and wind power can bring out better results than the traditional methods.

\section{Error analysis method}

The method can do error statistics for the predicted results at any time intervals. The error indicators should include root mean square error, average absolute error rate, and relative coefficient. The computational method for the indexes is as follows:

1) Average absolute error rate:

$\sum \mid$ predictedalue- actualvalue/numberof samplesinstalledcapacity

2) Root mean square error:

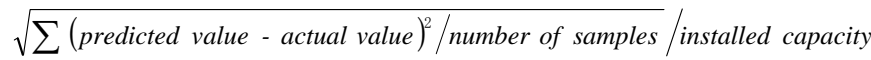

(2)

3) Relative coefficient:

|predicted value - actual value|/installed capacity

(3)

\section{SYSTEM DESIGN}

\section{A. Technical requirements for system}

Construction of wind power forecasting and application system is mainly used to help dispatch department to formulate power scheme, schedule operation plan and realtime control. In order to meet the demand, construction of wind power forecasting and application system must meet the following technical requirements:

For the technical requirement in time, the system must forecast one day before. Every day, forecast wind power of the next day $(0 \sim 24 \mathrm{~h})$ at 10:00, with time resolution $15 \mathrm{~min}$, used to develop power scheme, (0 6h) rolling forecast, for real-time monitoring of the dispatcher, (0 15min) ultrashort-term rolling forecast, for AGC control.

For the technical requirement in space, the system must forecast the output power of every farm, and the wind power in partial control area and the entire region. 
Also, the system must meet the relative technical requirements of automation; and have good interface with the EMS system.

\section{B. System architecture}

The wind power forecasting and application system is based on six technologies -- technology of acquisition and fusion about data from multi-system multi-type, GIS-based technology to process and display the integrated data, technology of information model, software developing technology, network communication technology, and information security technology. The system is divided into four layers: information presentation layer, business function layer, data exchange layer, physical support layer, as shown in Figure 2:

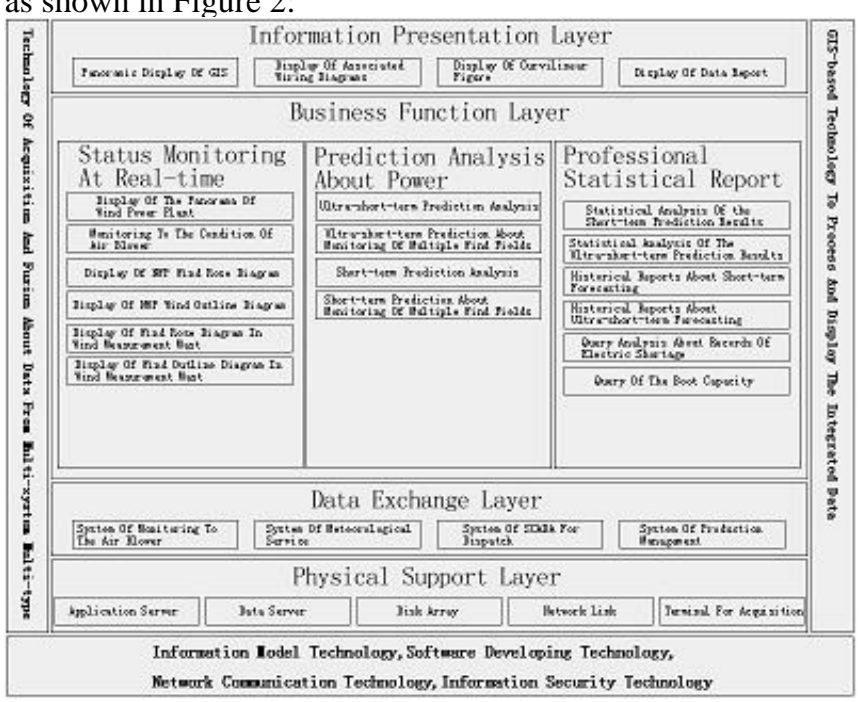

Figure 2. architecture of the system

\section{1) hysical support layer}

Physical support layer is to provide support at the level of hardware devices on the system, which should at least contain an application server for the deployment and publishing of service, a database server used to install the database and data warehouse, a set of RIAD(Redundant Array of Inexpensive Disks) for mass data storage, a set of network link used for data transmission and information exchange, terminal equipment for acquisition used for collection of data, and so on.

2) Data exchange layer

Data exchange layer is mainly used for data exchange and information-gathering with related business systems, to provide the good data support for the system. Construction of the system needs data about the state of blowers from the system of monitoring to the air blower, data about the meteorological information from the system of meteorological service, data dispatching load from the system of SCADA for dispatch, data about ledger of the equipment and operation and maintenance from the system of production management, and spatial information services for GIS services.

3) Business function layer
Business function layer is the core of building the system, which constructs the business model based on the research of wind power forecasting and application technology, enabling calculation, analysis of the mass information. As well, this layer establishes the association between different business data, enabling comprehensive analysis and display of integrated multi-system data. This layer contains three sections: status monitoring at real-time, prediction analysis about power, professional statistical report.

\section{4) Information presentation layer}

Information presentation layer is based on GIS platform in grid. This layer displays the system data visually through combination of field wiring diagram of wind farm, power flow charts, weather map and other types of thematic maps, and various curves graphics, reports of data, and other methods of presentation.

\section{Software functions}

Business function of wind power forecasting and application system can be concluded into 4 aspects: status monitoring at real-time, curve analysis, meteorological information display, and reports statistics. As shown in Figure 3:

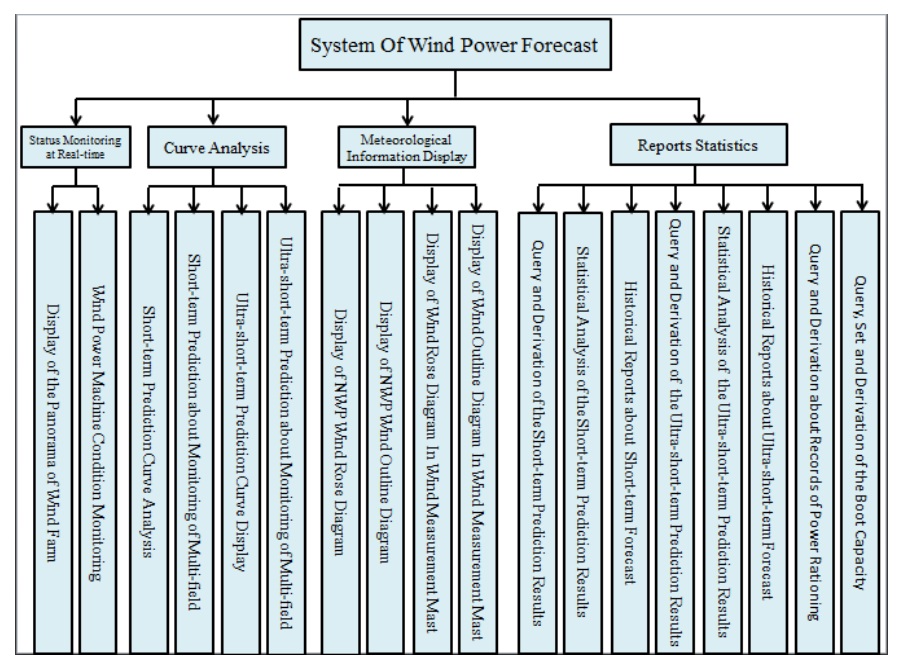

Figure 3. Function structure of the system

\section{1) Status monitoring at real-time}

Status monitoring at real-time is consists of display of the panorama of wind farm and distribution exhibits of the turbine. The panorama of wind farm shows the distribution of the wind farms within the region by the way of geographical distribution; the distribution exhibits of the turbine shows the distribution of turbines in specific farm by the way of geographical distribution.

\section{2) Curve analysis}

Display of curve includes short-term prediction curve analysis, short-term prediction about monitoring of multifield, ultra-short-term prediction curve display, ultra-shortterm Prediction about monitoring of multi-field. 


\section{3) Meteorological information display}

Meteorological information display includes display of NWP wind rose diagram, display of NWP wind outline diagram, display of wind rose diagram in wind measurement mast, and display of wind outline diagram in wind measurement mast.

\section{4) Reports statistics}

Reports statistics include query and derivation of the short-term prediction results, statistical analysis of the shortterm prediction results, historical reports about short-term forecast, query and derivation of the ultra-short-term prediction results, statistical analysis of the ultra-short-term prediction results, historical reports about ultra-short-term forecast, query and derivation about records of power rationing, query, set and derivation of the boot capacity.

Data statistics of historical data include statistics of completion, frequency distribution and the rate of change.

Data statistics of historical anemography and numerical weather forecast include statistics of completion, frequency distribution of wind speed and wind direction.

Parameters statistics of the operation in wind farm include statistics of output of plant, limited time for generation, maximum time of handling and occurrence, rate of simultaneousness, number of utilization hours, average loading rate. And the automatic generation of reports of specified format is supported.

\section{CONCLUSION}

This paper analyzed the problems grid-connected wind power faced, according to which proposed wind power forecasting system based on BP neural network. a proposal about a set of wind power forecasting and application system is presented, which is able to meet the needs of wind power forecasting and application in electric power enterprises.

\section{References}

[1] Han Shuang. Research on short-term prediction in wind farm. Beijing: North China Electric Power University, 2008.

[2] Lei Yazhou,Wang Weisheng, Yin Yonghua, Dai Huizhu. Analysis of the value of wind power on power system. Electrical Network Technology. 2002,2(5):10-14.

[3] Chi Yongning. Research on the stability when large-scale wind farms connected to the grid. China Electric Power Research Institute .2006.

[4] Fan Gaofeng, Wang Weisheng, Liu Chun. Wind power short-term prediction system based on artificial neural network. Electrical Network Technology. 2008, 32 (22) : 72-76.

[5] Hong Cui, Lin Weiming, Wen Buying. Overview on Prediction Methods of Wind Speed and Wind Power. Power System and Clean Energy. 2011, 27(1) : 72-76 . 